\title{
Preface
}

\section{Endocrine Pathology: Advances, Updates, and Diagnostic Pearls}

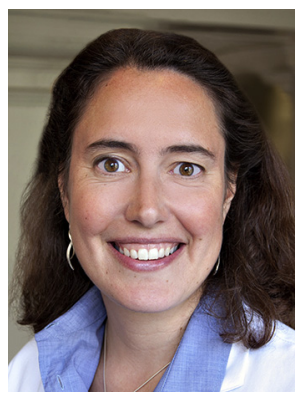

Justine A. Barletta, MD

Editor
It has been an exciting decade to practice endocrine pathology. Advances in our understanding of the molecular basis of thyroid tumors have changed the field. Recent iterations of molecular tests have further improved the triaging of patients with cytologically indeterminate nodules to surgery or surveillance. Insight into the molecular basis of thyroid tumors has also played a pivotal role in the reclassification of tumors, such as encapsulated follicular variant of papillary thyroid carcinoma, and is increasingly being used to refine risk stratification and inform treatment strategies. However, the advances in endocrine pathology are in no way limited to thyroid tumors. For example, virtually every year a new gene is found to be associated with the familial development of pheochromocytomas and paragangliomas. In addition, knowledge of the molecular underpinnings of pancreatic neuroendocrine neoplasms has impacted tumor classification.

This issue of Surgical Pathology Clinics is devoted to endocrine pathology. The issue is filled with articles outlining a practical approach to the diagnosis of an array of endocrine neoplasms. In addition, 4 articles written by experts in endocrinology, endocrine surgery, and oncology are meant to serve as a resource for pathologists to deepen their understanding of how patients with endocrine tumors are treated and how diagnostic parameters in our reports influence treatment decisions.
The first third of the issue is dedicated to thyroid pathology. Although the number of diagnostic entities in thyroid pathology is relatively small, diagnostic challenges arise due to pitfalls and controversies. The authors of the first article carefully review the many pitfalls in thyroid cytopathology (and how to avoid them). The next outstanding review not only provides an indepth discussion of prognostic parameters for differentiated thyroid carcinoma but also tackles some of the controversies, including what constitutes vascular invasion. After that, uncommon (but clinically important) thyroid entities are reviewed, and the molecular basis of thyroid tumors is addressed. Two articles devoted to the treatment of thyroid tumors (differentiated thyroid tumors and aggressive thyroid carcinomas) complete the thyroid section. The next group of articles focuses on adrenal pathology. Drs Sadow and Guilmette (who deserve special mention because they contributed 2 articles to this issue!) inform the reader of the frequent familial basis and current state of risk stratification of pheochromocytomas and paragangliomas. The pathology of the adrenal cortex is examined in the following article, with an expert review of algorithms used to distinguish benign from malignant adrenal cortical tumors. This section ends with an article covering the basics of treatment of adrenal cortical carcinoma. Parathyroid pathology is next considered. From there, the issue turns to gastroenteropancreatic 
(GEP) neuroendocrine tumors, with 2 outstanding reviews: the first provides a thorough overview of GEP neuroendocrine tumors at each site and updates the reader on tumor grading. The second article discusses treatment options for patients with these tumors. The issue concludes with a concise and practical guide to neuroendocrine tumors of the lung.

I would like to thank all of the authors who have contributed to this issue. I am grateful for the time they took from their exceedingly busy schedules to contribute thoughtful articles that are a true reflection of their expertise, insight, and passion for this field.

Justine A. Barletta, MD Chief, Endocrine Pathology Service

Department of Pathology Brigham and Women's Hospital Associate Professor of Pathology

Harvard Medical School 75 Francis Street Boston, MA 02115, USA

E-mail address: jbarletta@bwh.harvard.edu 